\title{
Alterstice
}

Revue internationale de la recherche interculturelle

International Journal of Intercultural Research

Revista International de la Investigacion Intercultural

\section{Diversité des modes matrimoniaux : vécu psychosocial des différents acteurs de systèmes familiaux polygames en milieu urbain au Sénégal}

\section{Marielle Bruyninckx, Dimitri Cauchie, Eugénie Dardenne et Marie Vande Ghinste}

Volume 7, numéro 2, 2017

Diversité(s) au coeur des politiques et des pratiques : entre l’Europe et l'Afrique

URI : https://id.erudit.org/iderudit/1052571ar

DOI : https://doi.org/10.7202/1052571ar

Aller au sommaire du numéro

Éditeur(s)

Alterstice

ISSN

1923-919X (numérique)

Découvrir la revue

Citer cet article

Bruyninckx, M., Cauchie, D., Dardenne, E. \& Vande Ghinste, M. (2017). Diversité des modes matrimoniaux : vécu psychosocial des différents acteurs de systèmes familiaux polygames en milieu urbain au Sénégal. Alterstice, 7(2), 77-89. https://doi.org/10.7202/1052571ar
Résumé de l'article

Cet article présente les résultats de deux recherches menées en milieu urbain à Dakar, auprès de familles polygames. Elles avaient pour objectifs d'étudier le vécu psychosocial de leurs acteurs et d'analyser les stratégies qu'ils mettent en oeuvre pour trouver leur place au sein de ces structures familiales complexes. Nos données montrent que ce régime matrimonial tend souvent à se

" simplifier » en évoluant vers une polygamie sans cohabitation. Les pratiques et le vécu polygamiques tendent donc à se diversifier dans un environnement social en évolution. Si parents et enfants évoquent des aspects positifs de leur vie, cette évolution n'empêche toutefois pas les difficultés relationnelles ni les stratégies d'affirmation de soi qui, chez les coépouses, varient en fonction de facteurs individuels (âge, rang matrimonial, niveau d'instruction, etc.). Cette compétition est un facteur de stress pour les maris, tenus au principe d'égalité de traitement. Enfin, un hiatus apparaît entre le discours que les enfants tiennent et leurs représentations de la vie familiale qui se dégagent grâce à nos autres instruments de recherche.
Droits d'auteur ( Marielle Bruyninckx, Dimitri Cauchie, Eugénie Dardenne et Marie Vande Ghinste, 2018
Ce document est protégé par la loi sur le droit d'auteur. L’utilisation des services d’Érudit (y compris la reproduction) est assujettie à sa politique d'utilisation que vous pouvez consulter en ligne.

https://apropos.erudit.org/fr/usagers/politique-dutilisation/ 


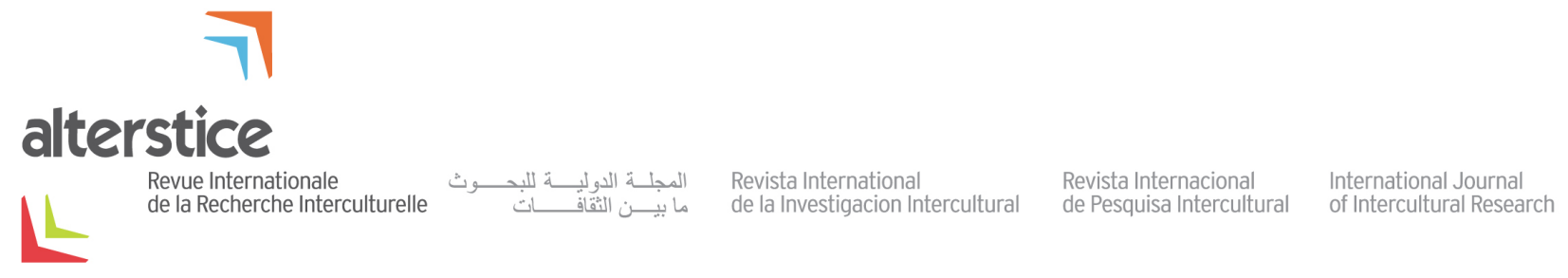

ARTICLE THÉMATIQUE

\title{
Diversité des modes matrimoniaux : vécu psychosocial des différents acteurs de systèmes familiaux polygames en milieu urbain au Sénégal
}

Marielle Bruyninckx ${ }^{1}$, Dimitri Cauchie ${ }^{1}$, Eugénie Dardenne ${ }^{1}$ et Marie Vande Ghinste ${ }^{1}$

\begin{abstract}
Résumé
Cet article présente les résultats de deux recherches menées en milieu urbain à Dakar, auprès de familles polygames. Elles avaient pour objectifs d'étudier le vécu psychosocial de leurs acteurs et d'analyser les stratégies qu'ils mettent en œuvre pour trouver leur place au sein de ces structures familiales complexes. Nos données montrent que ce régime matrimonial tend souvent à se "simplifier " en évoluant vers une polygamie sans cohabitation. Les pratiques et le vécu polygamiques tendent donc à se diversifier dans un environnement social en évolution. Si parents et enfants évoquent des aspects positifs de leur vie, cette évolution n'empêche toutefois pas les difficultés relationnelles ni les stratégies d'affirmation de soi qui, chez les coépouses, varient en fonction de facteurs individuels (âge, rang matrimonial, niveau d'instruction, etc.). Cette compétition est un facteur de stress pour les maris, tenus au principe d'égalité de traitement. Enfin, un hiatus apparaît entre le discours que les enfants tiennent et leurs représentations de la vie familiale qui se dégagent grâce à nos autres instruments de recherche.
\end{abstract}

\section{Rattachement des auteurs \\ ${ }^{1}$ Université de Mons, Belgique}

\section{Correspondance}

marielle.bruyninckx@umons.ac.be

\section{Mots clés}

Sénégal, diversités des modes matrimoniaux, systèmes familiaux polygames, stratégies d'affirmation de soi

\section{Pour citer cet article}

Bruyninckx, M., Cauchie, D., Dardenne, E. et Vande Ghinste, M. (2017). Diversité des modes matrimoniaux : vécu psychosocial des différents acteurs de systèmes familiaux polygames en milieu urbain au Sénégal. Alterstice, 7(2), 77-90. 


\section{Introduction}

La polygamie, mode matrimonial impliquant une organisation sociale reconnaissant les unions légitimes multiples et simultanées, est un phénomène extrêmement répandu dans le monde. La majorité de ces mariages polygames sont des mariages polygynes, reconnus légalement et encouragés par certaines pratiques religieuses. Attali et Bonvicini (2007) mentionnent que 45 pays, principalement en Afrique et en Asie et majoritairement de confession musulmane, autorisent la polygynie. L'hétérogénéité des contextes dans lesquels la polygamie s'organise, et des facteurs qui la font évoluer (culturels, sociaux) rend son vécu et les pratiques qu'elle implique de plus en plus diversifiés, et cette diversité croissante n'est encore que peu étudiée.

Au Sénégal, la polygamie, antérieure à l'Islam, est profondément ancrée dans l'organisation sociale : elle constitue traditionnellement un signe de réussite sociale et professionnelle pour l'époux et contribue à valoriser le rôle des femmes au travers de leurs fonctions d'épouse et de mère (Fainzang et Journet, 1988 ; Dial, 1999 ; Diop, 1985). La polygamie et les grandes familles sont ainsi source de sécurité et de prestige pour les membres du groupe (Lardoux et Van de Walle, 2003). En milieu rural, la polygamie a également une fonction économique importante puisqu'elle permet d'accroître la main d'œuvre pour le travail des terres (Adjamagbo et coll., 2006). Enfin, globalement, dans un contexte où la mortalité infantile reste relativement élevée, elle permet à certaines familles de s'assurer une descendance nombreuse en augmentant les chances de voir survivre les enfants, ce qui représentait une forme de sécurité sociale et économique pour les parents vieillissants (Cook et Kelly, 2006).

Si l'urbanisation, l'industrialisation et l'usage de nouvelles technologies en agriculture, facteurs de mutation de la société sénégalaise, laissaient présager l'amorce d’un déclin de la polygamie, celui-ci ne semble pas s'être produit (Chojnacka, 2000). De même, la régression annoncée de ce mode matrimonial en milieu urbain, notamment pour des raisons sociétales, ne s'est pas plus réalisée (Antoine, 2002; Dial, 2001). Il semble, au contraire, que ce mode de vie s'y diversifie davantage en raison de l'évolution de différents facteurs externes.

En effet, les résultats de l'Enquête Démographique et de Santé à Indicateurs Multiples de l'Agence Nationale de la Statistique et de la Démographie confirmaient déjà que la polygamie restait une pratique assez répandue, concernant $35 \%$ des femmes en union, et dont la forme la plus courante tendait davantage vers la bigamie (75 \% des unions polygames). Mais ils mettaient surtout en évidence « des écarts importants dans la fréquence de la pratique de la polygamie selon certaines caractéristiques socioéconomiques », telles le milieu (rural/urbain), la région, l'âge ou le niveau d'instruction (ANSD, 2012, p. 59). Le rapport relatif au Recensement Général de la Population et de l'Habitat, de l'Agriculture et de l'Élevage de 2013 confirme en outre que si « de nombreux auteurs prédisaient, dès les années soixante, une disparition progressive de la polygamie, notamment sous l'effet de I'urbanisation, de la scolarisation, de l'ouverture aux valeurs occidentales, etc. [...] force est de reconnaitre que la polygamie demeure plus que présente et intègre des milieux jusqu'ici insoupçonnés » (ANSD, 2014, p. 279-280) : on semble ainsi désormais " assister à une accommodation de plus en plus affirmée chez les femmes intellectuelles dans une société où le mariage est très valorisé » (p. 284).

La diversité accrue, et peut-être inattendue, des individus concernés par les unions polygames nous a donc mené à (re)questionner leurs expériences personnelles, psychologiques et sociales, au travers de deux études de terrain.

\section{Expériences psychosociales des acteurs des systèmes familiaux polygames}

\section{Évolution du fonctionnement polygamique des ménages}

Selon Fainzang et Journet (1988), le fonctionnement des ménages polygames est généralement basé sur la hiérarchie entre les sexes, qui induit que la femme doit obéissance et respect à son mari. En retour, ce mode matrimonial prévoit aussi l'égalité de traitement des coépouses, qui exclut tout favoritisme et contraint le mari à la justice distributive entre elles (Motahari, 2000). Les modes d'habitat des familles polygames sont divers: les coépouses peuvent partager une même maison ou avoir des logements différents. En milieu rural, la maison s'articule généralement autour d'un espace commun (la cuisine), chaque femme disposant de sa chambre et d'espaces personnels (sanitaires, jardin). En revanche, en milieu urbain, l'espace réservé à chaque épouse est plus restreint et peut parfois se limiter à une chambre (Fainzang et Journet, 1988).

Alterstice - Revue Internationale de la Recherche Interculturelle, vol. $7, n^{\circ} 2$ 
L'évolution sociétale, à laquelle est liée celle des régimes de nuptialité, a entraîné l'apparition de nouvelles modalités d'organisation (Chojnacka, 2000). En milieu urbain sénégalais, par exemple, la polygamie sans cohabitation devient plus fréquente: I'homme peut avoir plusieurs épouses vivant chacune dans leur propre maison. Mais, alors qu'elle permettait auparavant à l'homme de s'enrichir, notamment grâce à la main-d'œuvre gratuite que représentaient les femmes et les enfants, la polygamie n'est, dans ce contexte, plus possible que si l'époux est suffisamment aisé financièrement. Les conditions socio-économiques entraînent d'ailleurs parfois une réduction de la grande polygamie (trois femmes et plus) au profit de la bigamie (Antoine et Bocquier, 1997; Blanc et Gage, 2000).

\section{Vécu psychosocial des acteurs familiaux en situation de polygamie}

Les conséquences psychologiques et sociales de la polygamie sur ses acteurs ne peuvent pas s'appréhender en termes dichotomiques. La polygamie peut avoir des effets positifs comme négatifs sur les différents membres de la famille (Cook et Kelly, 2006).

Pour les hommes, outre le statut socioéconomique prestigieux qu'elle lui confère traditionnellement en tant que signe de de réussite, la polygamie peut devenir source de stress intense en raison des conflits entre coépouses, le climat familial tendu qui en découle pouvant même mener à des actes de violences intrafamiliales (Sawadogo, 2006). Par ailleurs, l'obligation de justice distributive peut être à l'origine d'importantes difficultés financières pour l'époux.

Pour les femmes, la polygamie permet de mieux protéger leur santé en leur donnant la possibilité d'espacer les grossesses (Sawadogo, 2006), et peut susciter un sentiment de soutien, voire de sororité, chez les coépouses, ce que certaines considèrent comme un réel avantage (Campbell et coll., 2005). Cependant, Al-Krenawi (2001) montre aussi que la vie en union polygame engendre parfois des conséquences négatives sur la santé mentale des femmes. Ainsi, les facteurs de compétition, de jalousie et de distribution inégale des ressources peuvent contribuer à susciter une certaine acrimonie entre les épouses et engendrer de nombreux conflits.

En ce qui concerne les enfants, Al-Krenawi, Graham et Slonim-Nevo (2002) ont montré qu'ils présentaient des niveaux de scolarité et d'estime d'eux-mêmes plus faibles que leurs homologues issus d'unions monogames. Ces résultats seraient, en partie, liés au statut socioéconomique moins élevé dont bénéficieraient certains enfants de familles polygames. Par ailleurs, certains de ces enfants soulignent le manque de rapports avec leurs parents, surtout avec leur père, ce qui les placerait dans un état d'insécurité émotionnelle et engendrerait davantage de difficultés à établir leur identité personnelle. Selon Ward (2004), l'isolement physique et social dont souffrent ces enfants peut avoir des répercussions sur leur scolarité (en particulier des difficultés d'adaptation scolaire, des rapports sociaux difficiles avec les professeurs et les autres élèves).

\section{Présentation de la recherche}

\section{Contexte, objectifs et principes de la recherche}

Au Sénégal, la construction du système matrimonial débute généralement par une union monogame et se complète, les années suivantes, par des unions additionnelles avec des femmes plus jeunes. L'Islam, religion majoritaire du pays, autorise un homme à contracter jusqu'à quatre unions, mais il arrive qu'il " hérite » des veuves de l'un de ses frères. Dans ce cas, le nombre d'épouses peut atteindre six ou sept (Lardoux et Van de Walle, 2003). Ce mode de vie polygame, bien établi socialement, est extrêmement répandu dans le pays. Particulièrement intéressés par cette question en milieu citadin, nous avons choisi la capitale, Dakar, pour mener deux recherches dont le but est de cerner, aussi objectivement que possible, l'expérience personnelle des acteurs de systèmes matrimoniaux polygames (les coépouses et les maris mais aussi les enfants issus des différentes unions) dans leur éventuelle diversité de ressentis, motivations et stratégies. En tant que chercheurs en psychologie, nous recevons, de manière neutre, la parole de chacun d'entre eux et nous nous efforçons de ne porter aucun jugement de valeurs sur un mode de vie qui n'est pas autorisé dans les sociétés européennes. Une période d'observation participante et de partage du quotidien nous a permis de gagner la confiance de chacun des sujets. La présentation des résultats 
obtenus repose au maximum sur la transmission fidèle des propos qui ont été récoltés lors de la recherche de terrain, que nous nous efforçons de ne pas interpréter de manière orientée.

\section{Questions de recherche}

Avec les adultes, nous avons tenté de répondre aux questions de recherche suivantes : quelles sont les motivations des hommes à devenir polygames ? Quel est le mode organisationnel mis en place par ceux-ci ? Quel vécu ont-ils de la situation ? Quel est le vécu des femmes et quelles sont les stratégies mises en œuvre par celles-ci pour trouver leur place au sein de l'union polygame ? Le vécu des femmes varie-t-il selon le rang de l'épouse ? Varie-t-il selon leur niveau d'éducation?

Pour les enfants, nos questions de recherche étaient les suivantes : quel est le vécu des enfants vivant au sein d'une famille polygame et quelles sont les relations entretenues avec ses différents membres ? Quelles représentations les enfants ont-ils de leur famille? Quels sont leurs projets familiaux et comment en conçoivent-ils le fonctionnement?

\section{Échantillons}

Nos données ont été recueillies auprès de 3 échantillons répondant à différents critères d'inclusion. Pour ceux des hommes (1) et des femmes (2), les répondants devaient vivre à Dakar dans une famille fonctionnant en polygamie, comprendre le français et être capables de s'exprimer dans cette langue. Les répondants constituant l'échantillon des enfants (3) devaient en outre être âgés d'au moins 6 ans et parler français (pour avoir une bonne compréhension de ce qui est demandé).

\section{Méthodologie et instrumentation}

Cette recherche est de type exploratoire et met en œuvre une méthodologie essentiellement qualitative, basée sur des études de cas. Le but est de répondre aux questions de départ afin de pouvoir émettre des hypothèses qui feront l'objet de recherches ultérieures. Elle se double d'une approche compréhensive car elle tente, au travers du discours des individus et des familles, de mettre en évidence la manière dont ils vivent la réalité du système familial. Pour ce faire, nous avons utilisé, avec les adultes, cinq outils : I'observation participante, le questionnaire anamnestique, le génogramme, l'entretien semi-directif et l'inventaire d'estime de soi de Coopersmith. Avec les enfants, nous avons utilisé l'observation participante, le questionnaire anamnestique, le génogramme, l'entretien semi-directif, le dessin de la famille dans trois déclinaisons ainsi que le test du système familial (FAST) de Gehring.

\section{L'observation participante}

Grâce à l'observation participante, le chercheur a pu s'intégrer à la vie des personnes, ce qui a donné lieu à des interactions sociales intenses au cours desquelles des données ont pu être collectées. Cette méthode, souvent utilisée en anthropologie et en sociologie, vise l'étude d'une société ou d'une communauté en partageant le mode de vie de ses membres, en se faisant accepter par eux et en participant aux activités des groupes. Cette technique nécessite que le chercheur fasse preuve de grandes capacités de prise de distance, notamment affective. Dans le cas de notre recherche, l'observation participante nous a permis de gagner la confiance des sujets et de mieux comprendre globalement les données récoltées. Elle ne donnera donc pas lieu à un point particulier dans l'analyse des résultats.

\section{Questionnaire anamnestique}

Le questionnaire anamnestique permet de recueillir des informations signalétiques (âge, origine ethnique, religion, nombre de coépouses, etc.) afin de pouvoir décrire correctement l'échantillon et de vérifier le respect de nos critères d'inclusion. 


\section{Le génogramme}

Le génogramme est une technique fréquemment utilisée par les psychologues en thérapie familiale systémique. Grâce au recueil d'informations sur les relations entre les membres de la famille, il permet d'établir un « arbre généalogique » de celle-ci et de bien comprendre la structure familiale afin d'en avoir une vision claire et rapide. II fournit, en effet, une représentation graphique synthétique favorisant la compréhension des systèmes familiaux parfois complexes. Cette représentation fait immédiatement apparaître, par des traits codifiés, les liens biologiques et légaux qui unissent les différents membres de la famille ainsi que ceux existant entre les différentes générations. Il complète avantageusement la récolte de données réalisée avec le questionnaire anamnestique.

\section{L'inventaire d'estime de soi (SEI) de Coopersmith}

L'inventaire d'estime de soi de Coopersmith permet de recueillir des données sur la perception et l'opinion qu'ont les personnes sur elles-mêmes. Il porte sur quatre domaines au travers d'autant d'échelles : une échelle générale (26 éléments), une échelle familiale (8 éléments), une échelle sociale ( 8 éléments) et enfin une échelle professionnelle (8 éléments). Au total, l'inventaire se compose de 58 éléments pour lesquels les sujets doivent indiquer une "ressemblance" ou non avec leur situation: les 50 précédemment cités et 8 éléments de " mensonge » dont les réponses ne sont pas comptabilisées dans les résultats totaux. Cette dernière échelle vise à déceler une éventuelle attitude défensive du sujet à l'égard du test ou un désir manifeste de donner une bonne image de soi. Les notes partielles s'obtiennent par simple addition des réponses positives. Outre ses qualités métrologiques, cet outil offre comme avantage une certaine rapidité dans l'administration ainsi que dans le dépouillement des données.

\section{L'entretien semi-directif}

L'entretien semi-directif a permis d'obtenir des informations plus ciblées sur le vécu des personnes rencontrées et de compléter les observations réalisées sur le terrain. Nous avons ainsi pu recueillir des données sur différentes thématiques tout en respectant la dimension interpersonnelle de la communication. En suscitant la parole spontanée du sujet sur un ensemble de thèmes, fixés à l'avance et consignés dans un guide, ce type d'entretien, par la souplesse qu'il garantit à l'échange, permet au chercheur d'orienter la récolte des données de manière naturelle et avec considération pour les propos qui lui sont adressés. Il permet de recueillir, notamment, des représentations culturelles, sans trop en contraindre l'expression. La grille d'entretien a été élaborée par nos soins et nous a servi de support afin de ne pas nous éloigner des objectifs de notre étude. Toutefois, l'ordre dans lequel les thématiques ont été évoquées a pu être modifié en cours d'entretien.

\section{Le dessin}

Le dessin est, pour le psychologue, une méthode projective grâce à laquelle on peut obtenir la représentation mentale et la connaissance qu'un individu a de son monde. II permet, par exemple, à l'enfant de s'exprimer plus facilement que par le biais de la parole. En effet, « le dessin d'enfant exprime bien autre chose que son intelligence ou son niveau de développement mental : une sorte de projection de sa propre existence et de celle des autres ou, plutôt, de la manière dont il sent exister lui-même et les autres » (Boutonier, 1953, p. 25). Dans le cas de notre recherche, nous avons souhaité utiliser cet outil dans la mesure où celui-ci s'avère particulièrement utile lorsque la verbalisation des affects est rendue difficile, voire impossible, en raison de diverses circonstances. En effet, nous avons très vite eu le sentiment qu'il était peu envisageable pour les enfants de critiquer ou d'émettre leur avis à propos du fonctionnement familial ou des choix réalisés par leur père en matière de conjugalité. Afin de bien comprendre les représentations que se faisaient les enfants de leur famille ainsi que leurs projets personnels, nous leur avons demandé de produire trois dessins, sur base de consignes différentes. Le premier devait représenter leur famille telle qu'elle était au moment de la recherche, le deuxième la famille idéale à laquelle ils souhaiteraient appartenir en tant qu'enfant, et le troisième la famille qu'ils désireraient fonder plus tard, quand ils seraient mariés. 
Le test du système familial (FAST) de Gehring

Le FAST (Gehring, 1992) est un outil de placement tridimensionnel qui permet le recueil d'informations au sujet de la représentation du système familial. Il est composé de figurines en bois, masculines et féminines, qui doivent être placées sur un damier. Le placement permet d'établir des hypothèses sur les relations familiales. Cet outil évalue la cohésion de la famille et la hiérarchie qui la caractérise. Cet outil permet de déterminer un type de structure relationnelle au sein de la famille et de ses sous-systèmes (parents, fratrie).

Planning de la recherche

L'ensemble de l'étude a été mené en suivant le protocole illustré sur la figure 1.

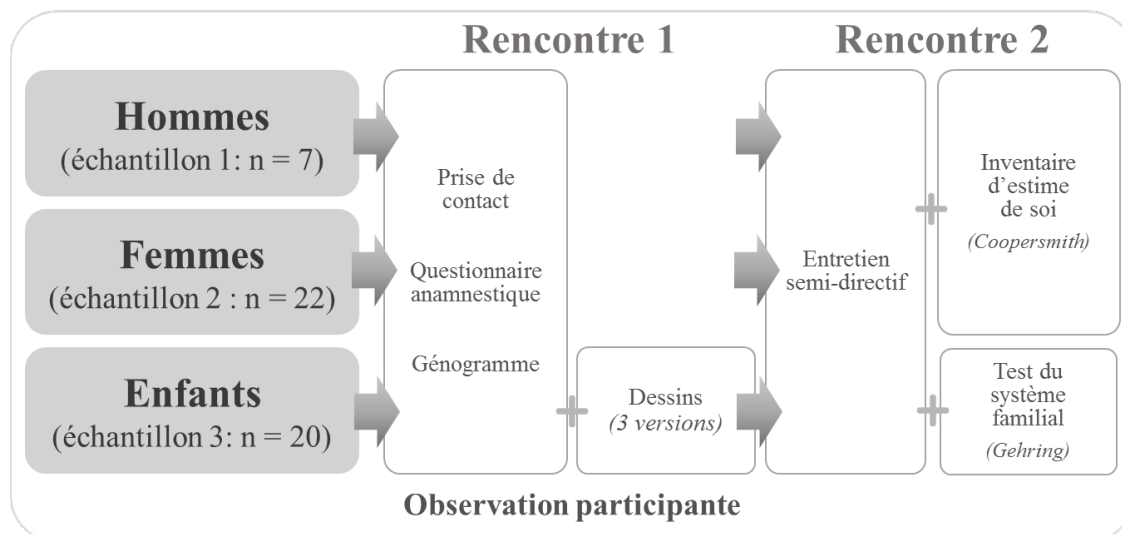

Figure 1 - Protocole de l'étude (échantillons et instrumentation associée)

\section{Présentation des principaux résultats}

\section{Résultats concernant les hommes}

Données anamnestiques et génogrammiques

Notre premier échantillon se compose de 7 hommes vivant en situation de polygamie dont l'âge moyen est de 47,6 ans (étendue de 37 à 49). Cinq sont Wolof, 1 est Lébou et le dernier Bambara. Tous sont musulmans.

Tous ont deux coépouses (respectivement âgées, en moyenne, de 38,4 pour la première et 29 ans pour la seconde). Parmi ces hommes, 6 ont opté pour le système de polygamie "séparée » où chaque épouse dispose de sa maison.

Analyse des entretiens : motivations à la polygamie

Les hommes de notre échantillon se disent tous favorables à la polygamie. Selon eux, ce mode matrimonial convient pleinement à la société sénégalaise, qui compte plus de femmes que d’hommes. La polygamie doit donc être considérée comme un service rendu à ces femmes qui peuvent ainsi trouver un foyer, un mari et fonder une famille. Pour nos 7 sujets, la polygamie est un privilège qui leur est accordé par l'Islam et « dont ils auraient tort de se priver » dans la mesure où il contribue à leur épanouissement sexuel. Un homme ayant de multiples partenaires aurait en effet une sexualité plus épanouie qu'un homme n'ayant des relations sexuelles qu'avec une seule femme. La polygamie serait, pour eux, un moyen d'institutionnaliser le fait que les besoins sexuels de l'homme sont plus importants que ceux de la femme. Néanmoins, d’autres motivations sont parfois énoncées. La polygamie garantit ainsi une descendance nombreuse qui assurera une certaine sécurité sociale et financière pour les vieux jours mais permet aussi d'afficher un certain statut et de perpétuer le clan dont on est issu (5 sujets sur 7). Trois hommes mettent surtout en lien la polygamie avec une certaine réussite sociale et professionnelle, qui est très valorisante pour eux au sein de leur entourage. Trois autres enfin estiment que la polygamie a été un moyen d'assagir ou de 
remotiver leur première épouse qui se montrait désobéissante ou avait tendance à se négliger. Ce mode matrimonial leur a permis de «pimenter » la vie conjugale devenue terne.

Analyse des entretiens : organisation et vécu psychosocial de la polygamie

Au sein de notre échantillon, 6 hommes sur 7 vivent en polygamie sans cohabitation. Pour eux, la séparation évite les conflits et la jalousie dans la mesure où les coépouses n'ont pas connaissance du comportement du mari avec chacune d'entre elles. Cette distance n'empêche pas pour autant la concurrence pour être la favorite car tous relèvent des astuces de séduction chez leurs différentes compagnes. Dans 2 cas, les hommes estiment que les relations entre leurs épouses sont ouvertement conflictuelles. Quant au seul sujet vivant sa polygamie en cohabitation, il estime que ce mode vie est plus confortable dans la mesure où il est transparent (« rien n'est caché ॥) et qu'il appartient à l'homme de bien gérer la situation afin de faire régner la paix dans son foyer. En ce qui concerne les finances, 6 hommes favorisent un traitement égalitaire de leurs épouses tandis que le dernier repartit le budget entre ses épouses selon le nombre d'enfants. L'apport financier important qu'il est nécessaire de rassembler à cet égard est pour eux une source importante de stress, dans la mesure où ils ne souhaitent pas donner une mauvaise image d'eux-mêmes. Le partage des moments de vie est assez régulé chez l'ensemble : la majorité d'entre eux (5/7) organisent ainsi des tours de deux jours afin de respecter un certain équilibre, un autre préfère des tours de quatre jours et le dernier se partage entre une épouse en ville où il travaille la semaine et une autre en campagne le week-end. L'ensemble des hommes interrogés disent utiliser des stratégies spécifiques pour alimenter la concurrence entre leurs épouses (attentions particulières, cadeaux...). Néanmoins, cette compétition peut aussi, à certains moments, devenir source de stress intense lorsque les rivalités et les jalousies suscitées deviennent ingérables (ex: lorsque les coépouses s'allient pour obtenir plus de leur mari). Une répartition traditionnelle, assez rigide, des genres est de mise dans l'ensemble des familles et l'autorité du mari est rarement remise en question.

\section{Analyse des inventaires d'estime de soi}

Les résultats à l'échelle générale de l'inventaire d'estime de soi laissent apparaître des scores se situant dans la moyenne, ou au-dessus de celle-ci, pour 5 hommes sur 7 qui, tous, possèdent une situation professionnelle avec des responsabilités importantes (ce qui se traduit par des scores élevés à l'échelle professionnelle) et une situation financière aisée. Leurs scores à l'échelle familiale traduisent une vie familiale heureuse, tout comme leurs résultats à l'échelle sociale, qui sont également très hauts. En revanche, les scores à l'échelle de mensonge sont bas dans la mesure où leur valorisation personnelle n'appelle ni désirabilité sociale supplémentaire ni attitude défensive à l'égard du test. Pour les 2 autres sujets, on peut mettre en lien les scores à l'échelle générale en-dessous de la moyenne avec le fait que ceux-ci n'ont pas fait d'études, sont analphabètes et n'ont pas de situation professionnelle qui les satisfait. Les rentrées financières sont insuffisantes pour combler tous les membres de la famille, ce qui se traduit par des scores bas aux échelles familiales et sociales. Un score élevé à l'échelle de mensonge traduit, en revanche, une attitude sans doute défensive à l'égard du test.

\section{Résultats concernant les femmes}

\section{Données anamnestiques et génogrammiques}

Notre deuxième échantillon comporte 22 femmes vivant en situation de polygamie dont l'âge moyen est de 41,7 ans (étendue de 24 à 59). Quatorze sont Wolof, 4 sont Peuls, 2 Sérères, 1 est Lébou et la dernière Mandingue. Toutes sont musulmanes.

Parmi elles, 4 sont les premières épouses d'une polygamie incluant deux femmes; 14 sont secondes et dernières épouses; 2 sont troisièmes et dernières épouses; 1 est deuxième épouse parmi 3 ; la dernière est deuxième épouse parmi 4. La majorité vit en polygamie sans cohabitation (14/22). 
Analyse des entretiens : vécu des femmes et stratégies mises en œuvre

Lors des entretiens semi-directifs, les femmes s'expriment fréquemment sur les conflits qu'elles rencontrent avec leurs coépouses. Elles décrivent les bagarres, les insultes mais aussi les pratiques de maraboutage que plusieurs d'entre elles utilisent. Elles soulignent la concurrence existant entre les coépouses et décrivent les nombreuses stratégies, notamment de séduction, mises en œuvre pour s'affirmer et susciter les faveurs du mari : cuisine, parfum, vêtements, relations sexuelles... Selon elles, cette concurrence est souvent entretenue par le mari qui en retire certains bénéfices personnels.

Une compétition au niveau de la reproduction est également souvent enclenchée pour s'attirer les bonnes grâces du mari. La pression sociale vécue à ce niveau n'est pas étrangère à cette course aux naissances. Dans certains cas, les femmes sont également touchées par la stérilité, ce qui aggrave la détresse ressentie. Selon certaines femmes, il semble que le chantage à la polygamie soit fréquemment utilisé par les hommes pour "assagir » ou pour " contrôler » leur(s) épouse(s) lorsque celle(s)-ci devien(nen)t trop difficiles à gérer.

\section{Analyse des entretiens : vécu des femmes selon leur rang d'épouse}

Ce sont surtout les premières épouses qui décrivent une souffrance intense car elles vivent souvent avec une grande stupéfaction le nouveau mariage du mari, et ce, malgré le caractère très répandu de la polygamie dans le pays. Nombreuses sont celles qui n'en avaient pas été averties à l'avance et qui n'imaginaient pas, après plusieurs années de relation monogame, vivre ce genre de modification de leur état matrimonial. Longtemps, elles ont pensé qu'elles ne seraient pas concernées par ce mode de vie, au contraire des autres femmes de leur entourage. Elles se sentent donc trahies, délaissées, abandonnées et décrivent l'annonce du nouveau mariage comme un événement d'une grande violence morale. Leur détresse affective fait également écho à l'évolution, ressentie comme négative, des rapports qu'elles entretiennent avec leur mari, avec lequel il leur est désormais impossible de vivre une relation de confiance. Nombreuses sont aussi celles qui déclarent avoir perdu le contrôle de leur vie, de n'être plus « dignes d'intérêt ", de ne pas "faire le poids " à côté de cette nouvelle épouse souvent plus jeune et plus jolie. Selon les moments, cela les rend tristes ou agressives. Dans certains cas, le mal-être est parfois aggravé par l'approche d'une ménopause qui, bientôt, ne leur permettra plus d'être fertiles. La plupart d'entre elles continuent néanmoins à tenter de trouver un sens à leur vie au travers de leurs (nombreux) enfants.

Les épouses suivantes semblent vivre la situation de polygamie avec moins de difficultés, notamment parce qu'elles savaient, dès le départ, que leur mari était déjà marié. II n’y a donc plus d’effet de surprise : c'est une union qu'elles ont accepté en toute connaissance de cause et avec laquelle elles tentent de s'accommoder malgré l'accueil peu empathique qui leur a été réservé par les épouses déjà présentes. Cela dit, à leur tour, elles regrettent de ne pas avoir été informées du mariage suivant et estiment, presque toutes, avoir ressenti un sentiment de trahison au moment où elles ont appris la nouvelle. En effet, l'arrivée de la coépouse suivante leur fait perdre le statut enviable de " dernière épousée ". Des sentiments négatifs et de tristesse s'installent chez ces femmes et on assiste, dans de nombreux cas, à des relations conflictuelles avec le mari et l'autre (ou les autres) épouse(s). En revanche, les dernières épousées bénéficient souvent d'une position dominante en tant que favorites du mari. Considérées comme jeunes et jolies, elles ont généralement une meilleure estime d’elles-mêmes.

\section{Analyse des entretiens : vécu des femmes selon leur niveau d'éducation}

Lorsque les femmes sont instruites, la vie polygame prend une tout autre figure. En effet, celles qui ont pu bénéficier d'une certaine instruction ont souvent un emploi et une relative autonomie financière qui les rendent moins dépendantes, moralement et financièrement, de leur mari. Au travers des vêtements et des parfums qu'elles peuvent s'offrir, elles disposent de davantage de moyens de séduction et leurs stratégies d'affirmation sont généralement plus réfléchies. Nos données soulignent qu'elles intellectualisent fréquemment leur discours à propos de leurs coépouses et qu'elles apparaissent, à leurs yeux, comme de redoutables rivales. Souvent, elles exigent de vivre dans un logement séparé de leurs coépouses et connaissent, au final, une situation proche de la monogamie qui leur convient parfaitement. Si elles apprécient les moments où leur mari est présent, elles savourent aussi ceux où il n'est pas là car elles ont alors un sentiment de liberté et d'autonomie. Plus éduquées, 
elles veillent aussi à limiter les naissances car elles sont sensibles à l'importance d'offrir une bonne éducation à leurs enfants.

\section{Analyse des inventaires d'estime de soi}

Lorsqu'on observe les résultats obtenus par nos sujets féminins pour l'échelle générale de l'inventaire d'estime de soi, on constate que 15 épouses ont obtenu un score se situant dans la moyenne tandis que 7 récoltent un score inférieur à celle-ci. Parmi elles, 5 sont des femmes habitant sous le même toit que leur coépouse, avec 3 d'entre elles qui n'ont pas fait d'études et sont sans emploi. Ces résultats confirment certains propos recueillis au cours des entretiens semi-directifs. En ce qui concerne l'échelle d'estime de soi familiale, 4 femmes ont obtenu un résultat élevé, 12 un résultat moyen et 6 un score inférieur à la moyenne. Parmi ces dernières, 3 ont été éduquées dans une famille monogame et vivraient donc plus difficilement ce mode de fonctionnement auquel elles n'ont pas été habituées dès le départ. Les résultats à l'échelle professionnelle sont caractérisés par une grande diversité et ne permettent pas la mise en lumière de tendances particulières. Enfin, il est intéressant de noter des scores élevés pour 13 femmes à l'échelle de mensonge, ce qui témoigne d’une désirabilité sociale forte, liée sans doute au souhait inconscient de répondre « au mieux » à ce test proposé par un chercheur étranger.

\section{Résultats concernant les enfants}

Données anamnestiques et génogrammiques

Notre troisième échantillon se compose de 20 enfants, 14 garçons et 6 filles, âgés en moyenne de 14 ans (étendue de 11 à 18). Dix-huit sont Lébous, deux sont Peuls.

La majorité vit dans un système familial où le père a deux coépouses (17/20), deux appartiennent à des familles avec trois coépouses et le dernier est issu d'une polygamie à quatre unions. Les parents de 11 de ces enfants ont opté pour le système de polygamie "séparée ».

\section{Analyse des entretiens semi-directifs}

Le discours des enfants apparaît assez ambigu et oscille entre le fait de décrire une situation de polygamie qui s'inscrit dans la normalité et une souffrance qu'ils ne souhaitent pas trop exprimer. Plusieurs ont ainsi du mal à se positionner par rapport à la tristesse de leur mère, aux absences du père mais aussi par rapport au rejet - vécu ou fantasmé - par la coépouse. Comme Devey (2000), nous relevons que les enfants grandissant dans des familles polygames sont soumis à plusieurs difficultés. Dans les familles où le mari exprime clairement sa préférence pour une épouse, l'enfant endosse un rôle de soutien pour sa mère délaissée et assume la souffrance de celle-ci. Les conflits de rivalité entre coépouses ont également un effet sur les enfants, par la distinction qu'ils opèrent entre ceux issus de lits différents. Les nouvelles formes de polygamie (sans cohabitation) ont l'avantage d'éviter ces disputes mais accentuent, pour ces enfants, le manque de relations avec leur père, plus souvent absent. L'image du père est dès lors ambiguë: il apporte le soutien matériel mais engendre un manque affectif qui fragilise les enfants; il est aimé et détesté à la fois mais ne peut faire l'objet de critiques de par le statut culturel qu'il détient dans les sociétés africaines. En ce sens, le recours à un outil d'investigation nécessitant peu l'usage de la parole peut s'avérer intéressant.

\section{Analyse des dessins : la famille actuelle}

À l'exception d'un sujet, tous les enfants représentent leur père dans leur dessin, et souvent en position centrale, ce qui traduit probablement la place importante qu'il occupe dans le système familial. Huit enfants, issus de polygamies sans cohabitation, dessinent leur famille en y incluant les différentes coépouses. Dans ce cas, le fonctionnement du système polygame semble évident, même si on remarque des liens distanciés entre certains personnages qui sont représentés de manière très éloignée sur la feuille (figure 2). Deux autres ne représentent aucune des femmes de la famille et les derniers omettent de représenter une des deux épouses (celle qui n'est pas leur mère). Seuls les neuf enfants issus de polygamies avec cohabitation dessinent la fratrie complète, mais parmi 
eux, cinq marquent néanmoins une séparation claire entre leur fratrie issue du couple parental et celle issue de leur père et d'une autre femme.

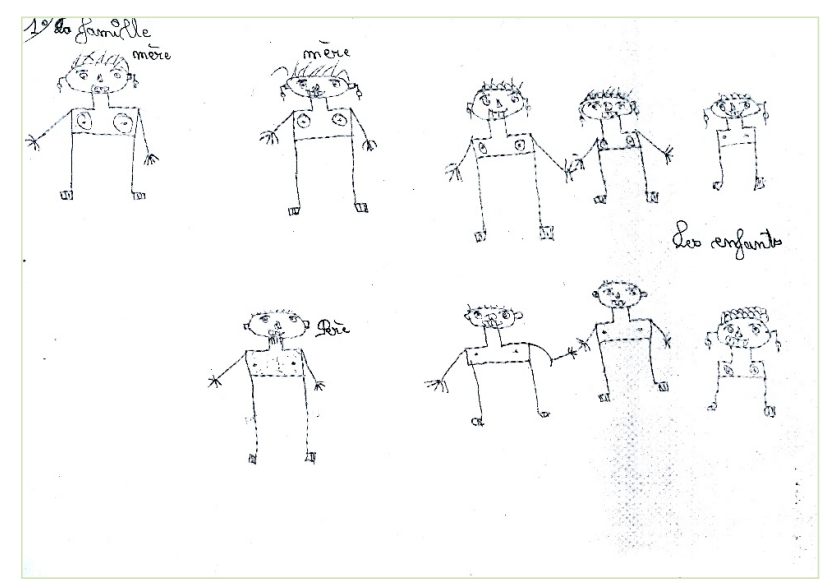

Figure 2 - Représentation de la famille actuelle (Abby, 13 ans)

Six sujets ne dessinent aucun des enfants à part eux-mêmes et les autres ne représentent que leur fratrie germaine. Ces dessins incomplets, ou marquant la distance entre les personnages, témoignent de difficultés de représentation de la famille dans son ensemble ou de tensions conflictuelles.

Analyse des dessins : la famille idéale

La majorité des enfants (15/20) représentent le père, les coépouses et tous les enfants, réunis en un seul ensemble (figure 3); 4 symbolisent uniquement leur famille nucléaire et un dessine toute sa famille mais avec des séparations nettes selon les sous-systèmes (épouse et coépouse). Clairement les enfants souhaitent donc une famille aux tensions relationnelles apaisées dans laquelle le père fait preuve de présence et occupe une position centrale.

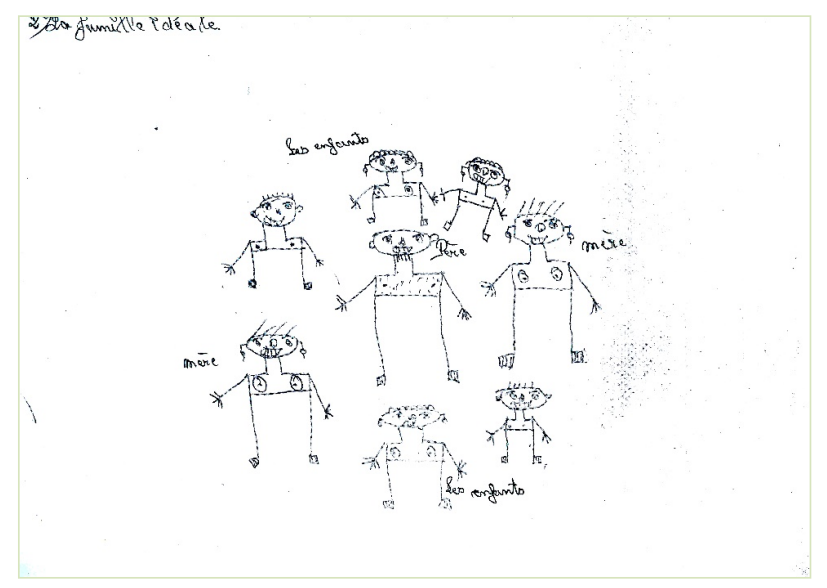

Figure 3 - Représentation de la famille idéale (Abby, 13 ans)

Analyse des dessins : la famille future

Seuls 4 enfants sur 20 souhaitent fonder une famille polygame; les autres préfèrent favoriser un schéma permettant un fonctionnement nucléaire avec un nombre d'enfants plus restreint (figure 4). On peut y voir le signe que certains se sentent peut-être un peu « perdus » dans cette grande fratrie qui est la leur et souhaite donner une place plus centrale à leurs propres enfants. 


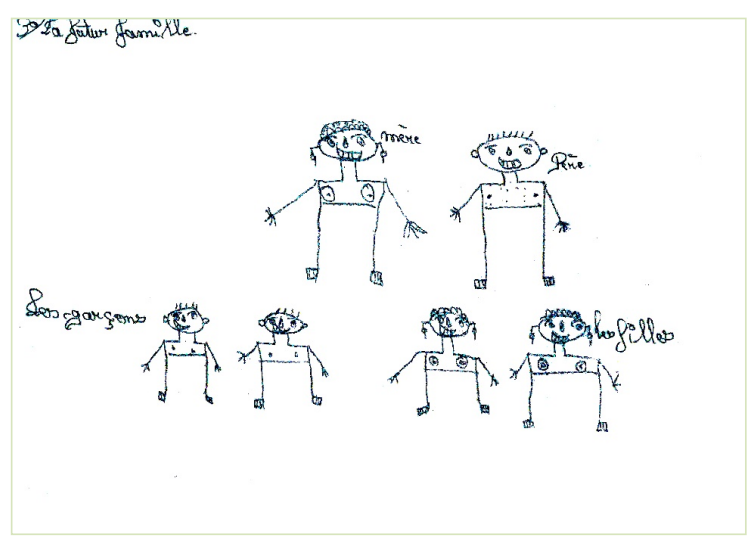

\title{
Figure 4 - Représentation de la famille future (Abby, 13 ans)
}

\author{
Analyse des tests du système familial (FAST)
}

De manière transversale, les résultats du FAST montrent un niveau de cohésion moyen au sein des familles mais avec des scores élevés pour la moitié des sujets en représentation conflictuelle, ce qui signifie qu'en cas de conflit, les membres de la famille tentent de se montrer solidaires afin de régler le problème. Lorsqu'on étudie les différents sous-systèmes familiaux de la famille polygame, on remarque une cohésion faible chez tous les sujets qui se traduit, dans les entretiens semi-directifs, par un désir de voir le couple parental plus uni que celui formé avec la coépouse. Le niveau de cohésion général relatif à la fratrie varie chez les sujets de moyen à faible. Les enfants vivraient donc leurs relations avec les frères et sœurs comme pouvant être améliorées. Au niveau des relations hiérarchiques, le test confirme, pour tous les sujets, la place prédominante du père dans le système familial ainsi que dans l'ensemble des sous-systèmes.

\section{Conclusion}

Les pratiques polygamiques au Sénégal ont évolué suite aux mutations sociétales que connaît le pays. Les structures familiales se transforment, se diversifient et se complexifient Le vécu de leurs membres aussi. De nombreuses recherches portant sur la polygamie en décrivent principalement ses effets négatifs, notamment sur les femmes. Moins d'études ont investigué les apports bénéfiques de la polygamie, ou se sont penchées sur les époux, ou sur les enfants. Notre recherche a ceci d'original qu'elle porte parallèlement sur des hommes, des femmes et des enfants afin d'étudier, en la recevant de manière neutre, la parole de chacun d'entre eux et de recueillir, sans porter de jugement de valeur, leurs vécus subjectifs et leurs perceptions du fonctionnement familial.

Pratique sociale soumise à des contraintes relationnelles presque sacrées et à de nombreuses convenances sociales, la polygamie semble bien installée au Sénégal, malgré les difficultés vécues au quotidien par les uns et les autres. Dans un pays où le divorce est très mal perçu et où les pressions familiales sont importantes, peu de couples tentent le parcours de vie monogame. Les pressions sociales et éducatives amènent de nombreuses femmes à survaloriser le mariage et la natalité, tout en s'inscrivant dans une idéologie de relative dépendance visà-vis des hommes. Toutefois, aujourd'hui, l'accès à la contraception est officiellement soutenu par le ministère de la Santé, qui voit l'accès au planning familial comme l'une des clés du développement du pays. Pour les hommes, la polygamie est une forme de réalisation sociale, une preuve de réussite qui les poussent à perpétuer ce mode matrimonial valorisé par la société dans laquelle ils vivent. Pourtant, s'il permet aux hommes de prendre plusieurs épouses, le Coran ne fait pas un dogme de la polygamie. Au contraire, il pose une série de conditions (notamment économiques) qui, de facto, limitent l'accès à cette forme de mariage et mettent sous pression les hommes concernés, dans un pays où la crise économique est bien présente. Si l'on remarque aujourd'hui une diversification relativement importante dans la mise en œuvre de ce régime matrimonial (la cohabitation entre les coépouses est de plus en plus évitée, au risque d'une certaine distanciation des liens familiaux traditionnels), l'observation de l'évolution de la diversité des pratiques polygames dans un pays où les jeunes, issus de ces familles polygames, sont de plus en plus connectés avec le monde occidental, sera très certainement intéressante à réaliser dans les années à venir. 
En effet, si l'on pourrait " penser que la scolarisation et le mode de vie à l'occidental vont réussir à cloisonner la pratique [polygamique] dans certains cercles réfractaires au changement" (ANSD, 2014, p. 284), les réalités semblent diverger de ces préjugés et, au contraire, suivre "une tendance qui va à rebours d'une idée reçue : la polygamie n'est plus réservée aux milieux populaires et ruraux" (Kane, 2018). Ces "nouvelles polygamies" méritent donc que la recherche les prenne en considération, dans le respect de la diversité des sociétés humaines et de leurs évolutions.

\section{Références bibliographiques}

Adjamagbo, A., Delaunay, V., Lévi, P. et Ndiaye ,O. (2006). Comment les ménages d’une zone rurale du Sénégal gèrent-ils leurs ressources ? Études rurales, 177, 69-90.

Agence Nationale de la Statistique et de la Démographie (2012). Enquête Démographique et de Santé à Indicateurs Multiples Sénégal (EDS-MICS) 2010-2011. Rapport final. Dakar : ANSD.

Agence Nationale de la Statistique et de la Démographie (2014). Recensement Général de la Population et de I'Habitat, de l'Agriculture et de l'Elevage. Rapport définitif. Dakar : ANSD.

Al-Krenawi, A. (2001). Women from polygamous and monogamous marriages in and out patient of psychiatric clinic. Transcultural Psychiatry, 38, 187-199.

Al-Krenawi, A., Graham, J. R. et Slonim-Nevo, V. (2002). Mental Health Aspects of Arab Israelic Adolescents from Polygamous Versus Monogamous Families. Journal of Social Psychology, 142, 446-460.

Antoine, P. (2002). Les complexités de la nuptialité : de la précocité des unions féminines à la polygamie masculine en Afrique (document de travail). Dakar : Développement et insertion internationale.

Antoine, P. et Bocquier, P. (1997). L'apport des enquêtes biographiques pour l'analyse de 30 ans d'évolution de l'emploi et du mariage : l'exemple de Dakar. Dans L'apport des collectes biographiques pour la connaissance de la mobilité : contributions aux débats. Paris : INED.

Attali, J. et Bonvicini, S. (2007). Amours : Histoires des relations entre les hommes et les femmes. Paris : Fayard.

Blanc, A. K. et Gage, A. J. (2000). Men, Polygyny, and Fertility over the Life-Course in Sub-Saharan Africa. Dans C. Bledsoe, S. Lerner et J. I. Guyer (dir.), Fertility and the Male Life-Cycle in the Era of Fertility Decline (p. 16387). Oxford : Oxford University Press.

Boutonier, J. (1953). Les dessins des enfants. Paris : Éditions du scarabée.

Chojnacka, H. (2000). Early marriage and polygyny: Feature characteristics of nuptiality in Africa. Genus, 3-4, 179208.

Campbell, A., Bala, N., Duvall-Antonacopoulos, K., MacRae, L., Paetsch J. J., Bailey, M., Baines, B., Amani, B. et Kaufman, A. (2005). La polygamie au Canada : conséquences juridiques et sociales pour les femmes et les enfants, Recueil de rapports de recherche en matière de politiques. Ottawa : Direction de la recherche Condition féminine Canada.

Cook, R. J. et Kelly, L. M. (2006). La polygynie et les obligations du Canada en vertu du droit international en matière de droits de la personne. Ottawa : Ministère de la Justice du Canada.

Devey, M. (2000). Le Sénégal. Paris : Karthala.

Dial, F. B. (1999). Divorce et promotion sociale et économique de la femme. Saint-Louis : Université Gaston Berger.

Dial, F. B. (2001). Le divorce, source de promotion sociale pour les femmes? L'exemple des femmes divorcées de Dakar et Saint-Louis (Sénégal). Colloque international « Genre, population et développement en Afrique. Abidjan : ENSEA-IFORD-INED-UEPA.

Diop, A. B. (1985). La famille Wolof. Paris : Karthala.

Fainzang, S. et Journet, O. (1988). La femme de mon mari, Anthropologie du mariage polygamique en Afrique et en France. Paris : L’Harmattan. 
Gehring, T. M. (1992). FAST : Test du système familial (trad. Arnone-Reitzle) [manuel]. Paris : Hogrefe.

Kane, C. (2018). Au Sénégal, la polygamie ne rebute plus les femmes instruites [En ligne]. Le Monde, 11 mai. http://www.lemonde.fr/afrique/article/2018/05/11/au-senegal-la-polygamie-ne-fait-plus-peur-auxfemmes-instruites_5297654_3212.htmI\#fQ8biLMxkspWcFxl.99

Lardoux, S. et van de Walle, É. (2003). Polygamie et fécondité en milieu rural sénégalais. Population, 58, 807-835.

Mc Goldrick, M. et Gerson R. (1990). Génogrammes et entretien familial. Montrouge : ESF Editeur.

Motahari, M. (2000). Les droits de la femme en Islam. Beyrouth : Al Bouraq.

Sawadogo, A. Y. (2006). La polygamie en question. Paris : L'Harmattan.

Ward, C. M. (2004). I Now Pronounce You Husband and Wives: Lawrence V. Texas and the Practice of Polygamy in Modern America. William and Mary Journal of Women and the Law, 11(1), 131-151. 